\title{
Computed tomography manifestations and pathological features of neuroendocrine carcinoma in uncommon sites
}

\author{
Fan Yang, Zhi Wen \\ Department of CT, Affiliated Cancer Hospital of Xinjiang Medical University, Urumqi, China \\ Contributions: (I) Conception and design: All authors; (II) Administrative support: Z Wen; (III) Provision of study materials or patients: Z Wen; (IV) \\ Collection and assembly of data: F Yang; (V) Data analysis and interpretation: F Yang; (VI) Manuscript writing: All authors; (VII) Final approval of \\ manuscript: All authors. \\ Correspondence to: Zhi Wen. Department of CT, Affiliated Cancer Hospital of Xinjiang Medical University, No. 789 East Suzhou Street, Xincheng \\ District, Urumqi 830011, China. Email: zw09dr_sea@163.com.
}

\begin{abstract}
Background: A neuroendocrine carcinoma is a malignant tumor that originates from the diffused neuroendocrine system (DNES). The present study aims to analyze the computed tomography (CT) manifestations and pathological features of neuroendocrine carcinomas in uncommon sites, in order to raise awareness of this disease.

Methods: The CT manifestations and pathological features of 29 cases of neuroendocrine carcinoma (confirmed by pathology) were retrospectively analyzed. The pathological results of these 29 cases were obtained through operations, and all cases underwent preoperative CT plain scanning and dynamic enhanced scanning.

Results: The neuroendocrine carcinoma sites were: the breast (five cases), the cervix (nine cases), the uterine body (one case), the gallbladder (two cases), the mediastinum (five cases), the pyelobladder (one case), the kidneys (one case), the bladder (two cases), the liver (one case), the pancreas (one case), and the ovaries (one case). The CT manifestations were as follows. Most of the carcinomas were solid, and in a mode of infiltrative growth. Poorly differentiated neuroendocrine carcinomas had blurred boundaries. Cystic necrosis could be observed in most lesions, and the calcifications were visible in some lesions. Of the 29 cases studied, 23 had moderate (>20 HU and <40 HU) or higher enhancement in the CT scans. Peripheral invasion, bone destruction and distant metastasis were more likely to be seen in these lesions.

Conclusions: The imaging manifestations of neuroendocrine carcinoma varies in uncommon sites. It would therefore be helpful to understand the diversity and imaging signs of neuroendocrine carcinoma, in order to improve diagnosis.
\end{abstract}

Keywords: Neuroendocrine carcinoma; pathology; X-ray computed tomography (X-ray CT)

Submitted Feb 21, 2020. Accepted for publication Sep 24, 2020.

doi: $10.21037 /$ tcr-20-1136

View this article at: http://dx.doi.org/10.21037/tcr-20-1136

\section{Introduction}

A neuroendocrine carcinoma is a malignant tumor that originates from the diffused neuroendocrine system (DNES). There was a time when neuroendocrine carcinoma was considered rare; however, with the wide application of pathological immunohistochemistry, the detection rate of neuroendocrine carcinoma has significantly increased, and the majority of scholars do not now regard it as a rare disease (1). Neuroendocrine carcinoma can occur in any part of the body, but is most common in the lungs and digestive system. The biological characteristics of neuroendocrine carcinoma in the lungs are the same as those of neuroendocrine carcinoma elsewhere in the body, with the characteristics of local recurrence and distant metastasis (2). To date, the literature on neuroendocrine 
carcinoma has focused on case reports of its pathological or clinical features, and few studies have analyzed the imaging features of neuroendocrine carcinoma in uncommon sites. In the present study, 29 cases of neuroendocrine carcinoma in uncommon sites were retrospectively analyzed. Then, the computed tomography (CT) manifestations and pathological features of neuroendocrine carcinoma were evaluated. Literature relating to both domestic and foreign cases was reviewed, and the imaging manifestations were summarized to improve the imaging diagnosis level of neuroendocrine carcinoma. We present the following article in accordance with the STROBE reporting checklist (available at http:// dx.doi.org/10.21037/tcr-20-1136).

\section{Methods}

\section{General data}

Over the period January 2014 to November 2019, 29 cases of neuroendocrine carcinoma from the Affiliated Cancer Hospital of Xinjiang Medical University were collected as study subjects. The inclusion criteria for patients were as follows: complete case data, diagnoses confirmed by surgical pathology, and complete preoperative CT data. Of the 29 cases of neuroendocrine carcinoma, 8 cases involved male patients and 21 cases involved female patients; patient ages ranged within $23-80$ years, with a median age of 55 . The sites of the carcinomas were as follows: five cases in the breast (two in the left breast and three in the right), nine cases in the cervix, one case in the uterine body, two cases in gallbladder, five cases in the mediastinum, one case in the pyelobladder, one case in the kidneys, two cases in the bladder, one case in the liver, one case in the pancreas, and one case in the ovaries. This study was conducted in accordance with the Declaration of Helsinki (as revised in 2013) and approved by the Ethics Committee of the Affiliated Cancer Hospital of Xinjiang Medical University (Approval No. K-2019052). Written informed consent was obtained from all participants.

\section{CT scanning method}

The GE Discovery HD 750 CT, the Somatom Definition AS 128 multi-slice spiral CT, the 64-row GE Lightspeed VCT, and the 16-row Philips Brilliance Big Bore multi-slice spiral CT were used to perform plain CT scans and dynamic enhanced scans. The parameters were used: slice thickness of $5 \mathrm{~mm}$, slice interval of $5 \mathrm{~mm}$, thin-slice reconstruction of
$1.25 \mathrm{~mm}$, tube current of $250 \mathrm{mAs}$, tube voltage of $120 \mathrm{kV}$, and display field of view (DFOV)n of $350 \times 350 \mathrm{~mm}$. For the enhanced scan, a high-pressure syringe was used to inject $100 \mathrm{~mL}(300 \mathrm{mgI} / \mathrm{mL})$ of non-ionic contrast agent into the elbow vein with a flow velocity of $3 \mathrm{~mL} / \mathrm{s}$.

\section{Diagnostic criteria and analysis}

All cases were reviewed by three experienced senior attending radiologists. In the case of any disagreement, a consensus was reached following consultation. The CT manifestations of the neuroendocrine carcinoma in different sites were analyzed, with analysis including: (I) boundary of the tumor; (II) morphology of the tumor; (III) existence of calcification; (IV) degree and mode of tumor enhancement; (V) relationship between the tumor and adjacent organs, and distant blood metastasis; and (VI) status of the peripheral lymph nodes. With pathology as the gold standard, the immunohistochemical analysis was performed by two pathologists.

\section{Results}

\section{Sizes, locations, and CT manifestations of the tumors}

Of the 29 cases confirmed by operation and pathology, 24 cases were single onset, while 5 cases were multiple in origin. Furthermore, 4 cases had a cystic solid mass, 2 cases had irregular thickening of the wall, and 23 cases had solid masses. The largest mass was approximately $10.5 \mathrm{~cm} \times$ $8.4 \mathrm{~cm}$ in size, and was located in the head of the pancreas, while the smallest measured approximately $1.0 \mathrm{~cm} \times 1.5 \mathrm{~cm}$, and was located behind the right papilla.

There were five cases of breast carcinoma (two of the left breast, and three of the right). Three cases were single onset, while two had multiple origins located in the upper quadrant of the right breast and behind the nipple. Macular calcification was visible in two cases. In addition, three cases were lobular in shape and had an obvious enhancement, while two cases had a blurred border, which mainly manifested as marginal enhancement and central necrosis (Figure 1). In addition, one case had skin invasion, two cases had clavicular region and axillary lymph node metastasis, and one case had lumbar, liver, and right lung metastasis.

There were nine cases of cervical tumors, and one case of uterine body lesions. Two cases involved tumors that were limited to the local cervix, seven cases involved invasion to the lower part of the uterine body or vagina, two cases 


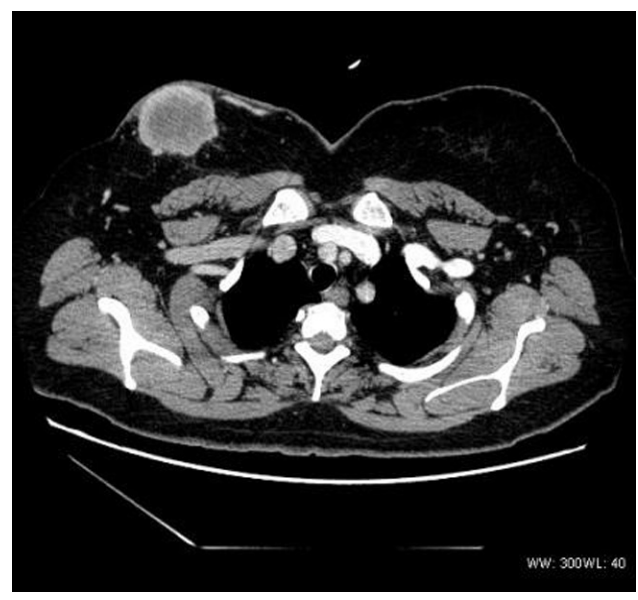

Figure 1 A 44-year-old female with a pathological diagnosis of neuroendocrine carcinoma. The CT revealed an irregular soft tissue mass in the upper quadrant of the right breast, which was lobulated in shape, with central necrosis and a mainly marginal enhancement. CT, computed tomography.

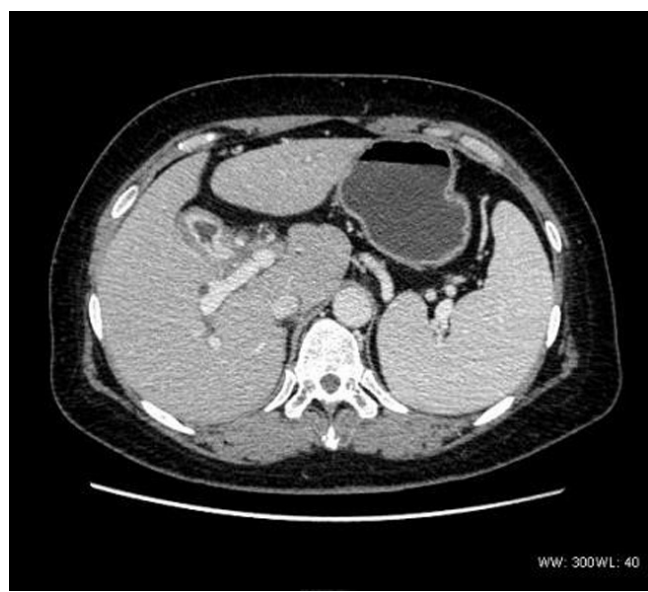

Figure 2 A 55-year-old female with a pathological diagnosis of neuroendocrine carcinoma. The gallbladder wall presented with irregular thickening and an obvious enhancement. The tumor invaded to the serous layer.

had lung and liver metastasis, and one case had pelvic and retroperitoneal lymph node metastasis.

In the two gallbladder tumor cases, the wall was irregular in shape and thickened; both were significantly enhanced (Figure 2). One case had hilar lymph node metastasis, and one case presented intrahepatic bile duct expansion, in which the tumor had invaded the serosa layer, together with nerve invasion.

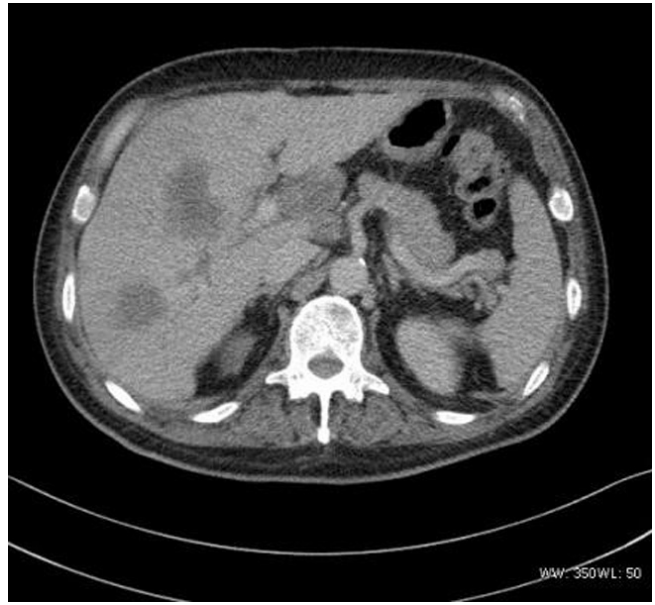

Figure 3 A 59-year-old male with neuroendocrine carcinoma of the anterior mediastinum, multiple hepatic metastasis, and multiple lymphadenopathies of the hilar region.

The density was uneven in the five mediastinum tumor cases, which included one case with patchy vcalcification. Four cases had uneven enhancement after the dynamic enhanced scan, and one case exhibited obvious enhancement. All five cases had lymph node metastasis. Furthermore, one case had multiple metastases of the bilateral lungs, subpleural, liver, and bone (Figure 3), and one case had involvement of the left and right pulmonary arteries and superior vena cava. In two cases the metastasis involved the pericardium and mediastinum pleura.

The pyelobladder tumor case had multiple masses with punctate calcification and moderate enhancement.

The renal tumor case revealed a moderate heterogeneous enhancement, blurred boundary with the renal pelvis, and thickening of the perirenal fascia (Figure 4).

There were two bladder tumor cases, which included one case with infiltrative growth and slight enhancement, and one multiple-onset case with lobulated mass, obvious enhancement, and lumbar and bilateral adrenal metastasis. Both cases had pelvic lymph node metastasis.

In the hepatic tumor case, the tumor was irregular in shape, with a blurred boundary and obvious marginal enhancement.

The single pancreatic tumor contained cystic and solid components, with local separation and spotty calcification, together with continuous and obvious uneven enhancement (Figure 5).

The ovarian tumor case presented with "honeycombed" changes (Figure 6), with obvious uneven enhancement and 


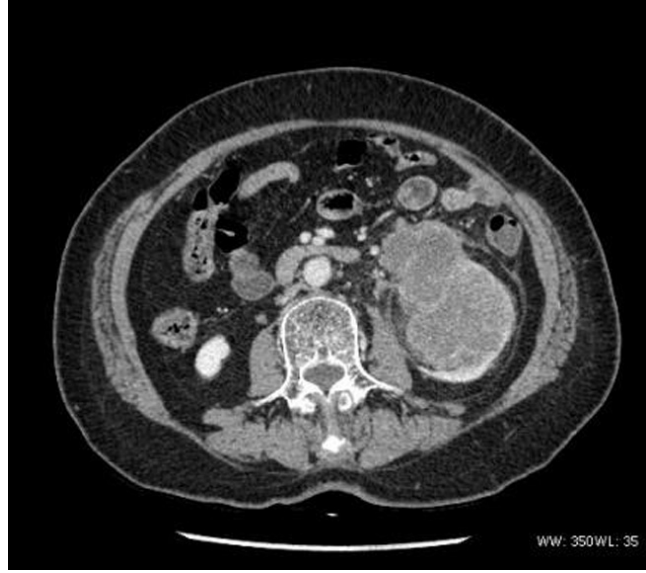

Figure 4 A 70-year-old female with a pathological diagnosis of neuroendocrine carcinoma. The irregular soft tissue mass of the left kidney, with a blurred boundary with the renal pelvis, and thickening of the perirenal fascia.

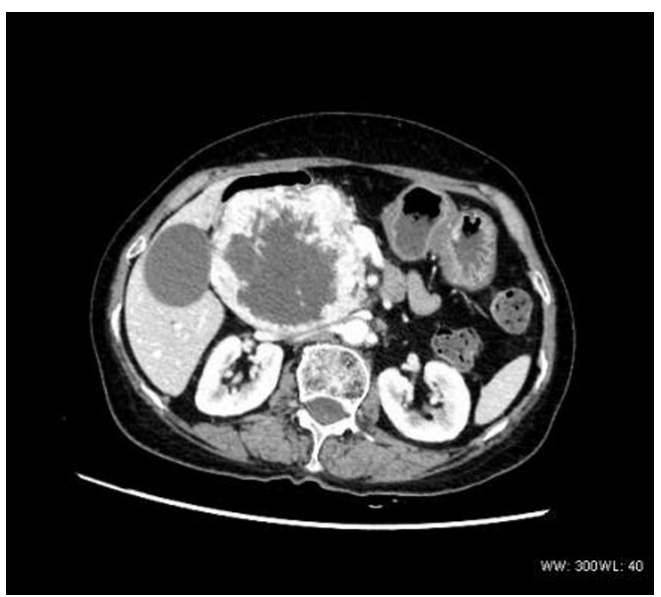

Figure 5 A 68-year-old female with a pathological diagnosis of neuroendocrine carcinoma. The tumor mass was located at the head of the pancreas, with most of the cystic lesions and necrosis located in the center. The CT revealed a continuous and obvious non-uniform enhancement, with a thick vascular shadow around and inside the mass. CT, computed tomography.

bone metastasis.

\section{Pathological manifestations and immunobistochemistry}

All cases in the present study were diagnosed as neuroendocrine carcinoma by routine pathology and immunohistochemistry. The pathological features were as follows. The tumor cells were polygonal, oval, or

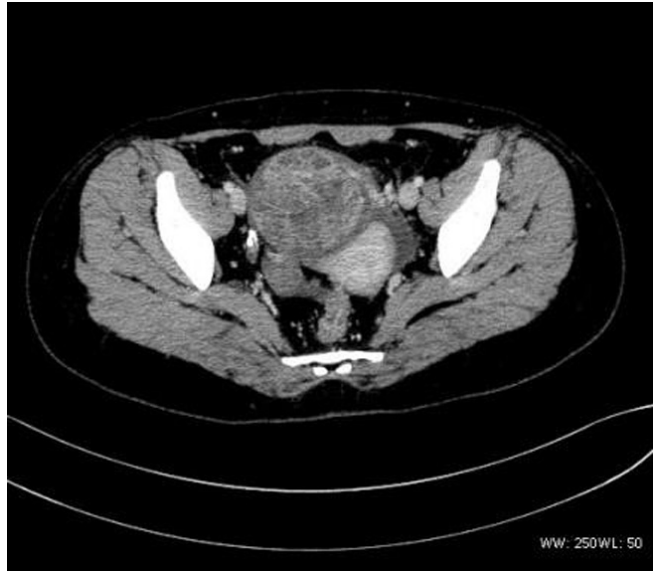

Figure 6 A 33 -year-old female with small cell carcinoma. The round tumor in the left ovary, with a clear and regular boundary, and local "honeycomb" changes.

fusiform in shape, rich in cytoplasm, and eosinophilic. The immunohistochemistry results were as follows. For 20 cases, Ki-67 $\geq 50 \%$ (positive), and for 9 cases, Ki-67 $<50 \%$ (positive). Of the 29 cases, 22 were positive for chromogranin A (CgA), 1 case was weakly positive for $\mathrm{CgA}, 1$ case was focally positive for $\mathrm{CgA}$, and 5 cases were negative for CgA. All 29 cases were positive for synapsin (Syn), with Syn (small focal positive) in 1 case, and Syn (partial positive) in 1 case. Out of 23 cases tested for CD56, 17 were positive for CD56, and 6 were negative. Out of 19 cases tested for the tissue keratin polypeptide (CK), 14 were positive. Only four cases were tested for the neuron specific enolase (NSE); two were positive.

\section{Discussion}

\section{The origin and clinical and pathological characteristics of neuroendocrine carcinoma}

The mechanism of neuroendocrine carcinoma remains unclear. Of the 29 patients in the present study, 8 were male and 21 were female, i.e., there were more females than males. The onset age of these patients ranged within 23-80 years; the median age was 55 . There were no specific symptoms and signs in patients after carcinoma onset. Some patients with small cell cancers may have carcinoid syndromes, such as skin flushing, asthma and diarrhea. In general, tumor cells originate from amine precursor uptake and decarboxylation cells in the endocrine system, or argyrophil cells in the digestive tract and bronchial 
mucosa glands. Approximately $85 \%$ of these are located in the stomach, intestine and pancreas (3), with fewer cases in other (uncommon) sites of the body. It has been reported that neuroendocrine carcinoma accounts for approximately $0.3-0.5 \%$ of breast cancers (4), approximately $0.9-1.5 \%$ of cervical tumors (5), and $2 \%$ in gallbladder tumors (6). Since there are few reports and few cases of neuroendocrine carcinoma in uncommon sites, standardized diagnosis and treatment guidelines are still lacking (7).

This type of tumor, also known as carcinoid or argyrophil tumor, is uncommon, with an incidence of 1.5 per 100,000 (8); however, epidemiological surveys from the United States, Europe, and Japan reveal that its incidence is increasing annually (9). The pathological features of this tumor are as follows. The tumor cells are small and uniform in size $(10,11)$. The nucleus is mostly round with a sunken nuclear membrane. The heterochromatin in the nucleus accumulates near the nuclear membrane (5), and there are many mitoses. There are characteristic endocrine granules in the cytoplasm. The hormones in mature granules have biological activity, while those in immature granules contain hormone precursors with low biological activity (11). The cells are positive in silver staining. Immunohistochemical markers include markers for neuroendocrine tumors and epithelial tumors: CgA, Syn, CD56, NSE, CK, etc.; diagnosis can be confirmed with at least two positive stains. Syn and $\mathrm{CgA}$ are highly specific neuroendocrine markers, and are of great value in the diagnosis of this kind of tumor (11). In the present study, all 29 patients were positive for Syn. However, only 24 of the 29 were positive for $\mathrm{CgA}$. Further tests were conducted: 23 patients were tested for CD56, with 17 positive results; 19 patients were tested for CK, with 14 positive results; and 4 patients were tested for NSE, with 2 positive results.

\section{CT manifestations and the pathological basis of neuroendocrine carcinoma}

Having summarized the CT features of neuroendocrine carcinoma in the present study, the investigators suggest that neuroendocrine carcinoma in uncommon sites has the following CT features:

(I) Mode of growth: Among the 29 cases in the present study (with the exceptions of one case of neuroendocrine carcinoma that originated from the ovary, one case of pancreas origin, and a small number tumors of breast origin that manifested as expansive growth), the growth mode of the carcinomas was mainly invasive growth, which is consistent with the literature (12). In the present study, four cases presented as multiple onset.

(II) Density, enhancement form, and degree of tumor differentiation: Most of the tumors were solid, with even or uneven density. Necrosis, cystic change, and spot or patchy calcification could be observed in some tumors $(13,14)$. Some of these tumors presented with even enhancement, while some presented with marginal enhancement. Four patients had cystic solid lesions, and one patient presented with "honeycomb" ovarian lesions and obvious enhancement after the enhanced scan (magnitude of enhancement: $>40 \mathrm{HU}=$ obvious enhancement, $>20 \mathrm{HU}$ and $<40 \mathrm{HU}=$ moderate enhancement, and $<20 \mathrm{HU}$ $=$ mild enhancement). In some tumors in the mediastinal and pancreas, there were thick and tortuous vascular shadows, which is consistent with the description in some domestic studies $(15,16)$. In the present study, fourteen patients presented with obvious enhancement, nine patients presented with moderate enhancement, and six patients presented with mild enhancement. These different enhancement patterns in the tumors might be correlated to the different pathological types, the proliferative activity of cancer cells, and the different sites of origin. Ki-67 is used to determine the proliferation activity of cancer cells. In the present study, 20 patients had Ki- $67 \geq 50 \%$ (positive), suggesting that the proliferation of neuroendocrine carcinoma cells was more active. Furthermore, it could be observed that multifocal necrosis was present, and in imaging, the lesions mostly presented with uneven enhancement, or the central area necrosis presented with a mainly marginal enhancement.

(III) Morphology and boundary: Most of the patients in the present study had irregular tumors with a lobulated shape and a blurred boundary; tumors with clear boundaries were highly differentiated.

(IV) Adjacent invasion and remote metastasis: Neuroendocrine carcinoma of the uterus, breast, kidneys, and bladder often presents with regional lymph node metastasis, or multiple metastases of the liver, lungs and bone. Mediastinal neuroendocrine carcinoma infiltratively grows 
along the space between the large blood vessels, and closely or indistinctly grows with the large blood vessels and pericardium at the bottom of the heart. In the present study, five patients with mediastinal neuroendocrine carcinoma had lymph node metastasis, and four patients had Ki-67 >50\% (positive). Of these latter four patients, one patient had multiple metastases of the lungs, subpleural, liver, and bone; one patient had metastasis that involved the left and right pulmonary arteries and superior vena cava; and two patients had metastasis that involved the pericardium and mediastinum pleura. Of these five patients, only one patient had Ki-67 $=20 \%$ (positive), and this patient's tumor presented with a regular appearance, uniform density and clear boundary, but with no surrounding invasion. This was very difficult to diagnose before operation.

\section{Conclusions}

Neuroendocrine carcinoma is a type of malignant tumor that can manifest in all parts of the body, with a variety of CT manifestations. Although there is a lack of high specificity, there are still many common characteristics. Through analysis of the CT and pathological features, the present study aimed to improve the understanding of neuroendocrine carcinoma, with positive significance for clinical treatment and prognostic evaluation. There are, however, some limitations to the present study. As a retrospective analysis, the sample size was small. In future studies, it will be necessary to expand the sample size, to explore the commonalities of images and of clinical and pathological characteristics of neuroendocrine carcinoma in different parts of the body, to aid diagnosis and prognostic evaluation of this disease.

\section{Acknowledgments}

Funding: This study was supported by Xinjiang Uygur Autonomous Region Health and Scientific Research Project for Young Medical Talents: Application of imaging omics in evaluating the efficacy of lung cancer immunotherapy (No. WJWY-202033).

\section{Footnote}

Reporting Checklist: The authors have completed the
STROBE reporting checklist. Available at http://dx.doi. org/10.21037/tcr-20-1136

Data Sharing Statement: Available at http://dx.doi. org/10.21037/tcr-20-1136

Conflicts of Interest: All authors have completed the ICMJE uniform disclosure form (available at http://dx.doi. org/10.21037/tcr-20-1136). The authors have no conflicts of interest to declare.

Ethical Statement: The authors are accountable for all aspects of the work in ensuring that questions related to the accuracy or integrity of any part of the work are appropriately investigated and resolved. This study was conducted in accordance with the Declaration of Helsinki (as revised in 2013) and approved by the Ethics Committee of the Affiliated Cancer Hospital of Xinjiang Medical University (Approval No. K-2019052). Written informed consent was obtained from all participants.

Open Access Statement: This is an Open Access article distributed in accordance with the Creative Commons Attribution-NonCommercial-NoDerivs 4.0 International License (CC BY-NC-ND 4.0), which permits the noncommercial replication and distribution of the article with the strict proviso that no changes or edits are made and the original work is properly cited (including links to both the formal publication through the relevant DOI and the license). See: https://creativecommons.org/licenses/by-nc-nd/4.0/.

\section{References}

1. Yao JC, Hassan M, Phan A, et al. One hundred years after "carcinoid": epidemiology of and prognostic factors neuroendocrine tumors in 35825 cases in the Unit States. J Clin Oncol 2008;26:3063-72.

2. MacIntosh PW, Jakobiec FA, Stagner AM, et al. High grade neuroendocrine neoplasm of the antrum and orbit. Surv Ophthalmol 2015;60:486-94.

3. Zhang L, Cheng HY, Long XA, et al. CT and MRI findings of primary hepatic carcinoid tumor. Chin J Radiol 2010;44:407-10.

4. López-Bonet E, Alonso-Ruano M, Barraza G, et al. Solid neuroendocrine breast carcinomas: incidence, clinicopathological features and immunohistochemical profiling. Oncol Rep 2008;20:1369-74.

5. Margolis B, Tergas AI, Chen L, et al. Natural history 
and outcome of neuroendocrine carcinoma of the cervix. Gynecol Oncol 2016;141:247-54.

6. Chen C, Wang L, Liu X, et al. Gallbladder neuroendocrine carcinoma: report of 10 cases and comparison of clinicopathologic features with gallbladder adenocarcinoma. Int J Clin Exp Pathol 2015;8:8218-26.

7. Wang S, Liu Z. Neuroendocrine carcinoma of the breast. Chin J Pract Surg 2013;33:238-40.

8. Dogra VS, Poblete J. Metastatic carcinoid tumor in the liver. J Clin Ultrasound 1993;21:639-41.

9. Bai B, Cang SD, Kan YZ, et al. Epidemiological and pathological analysis of 218 cases of neuroendocrine neoplasm. J Mod Oncol 2019;27:2391-4.

10. Hsueh C, Tan XD, Gonzalez-Crussi F. Primary hepatic neuroendocrine carcinoma in a child. Morphologic, immunocytochemical, and molecular biologic studies. Cancer 1993;71:2660-5.

11. Liu FH, Liu TH. Neoplastic pathology. Beijing: Peking

Cite this article as: Yang F, Wen Z. Computed tomography manifestations and pathological features of neuroendocrine carcinoma in uncommon sites. Transl Cancer Res 2020;9(11):69126918. doi: $10.21037 /$ tcr-20-1136
Union Medical College Union Medical University Press, 1997;1379-91.

12. Qu J, Yuan X, Zhao W, et al. CT and MRI manifestations of rare neuroendocrine cancers. Radiologic Practice 2019;34:1137-41.

13. Wang LX, Liu K, Lin GW, et al. Primary hepatic neuroendocrine tumors: comparing CT and MRI features with pathology. Cancer Imaging 2015;15:13.

14. Zhao CY, Hu JB, Liu CH, et al. CT diagnosis and differential diagnosis of primary hepatic neuroendocrine carcinoma. J Med Imaging 2018;28:1134-7.

15. Chen Zhu, Xiao EH. Dynamic Contrast Enhanced Computed Tomography and Pathologic Features of Hepatic Neuroendocrine Carcinoma. J Clin Radiol 2015;34:1766-71.

16. Xue P, Shi DP, Zhang SJ, et al. CT manifestations of the extra-pulmonary neuroendocrine carcinoma. J Prac Radiol 2012;28:393-433. 